\title{
Eye Drop Comfort Grade
}

National Cancer Institute

\section{Source}

National Cancer Institute. Eye Drop Comfort Grade. NCI Thesaurus. Code C147474.

The position on a scale of intensity, amount or quality of eye drop comfort. 